$15 *$ Corresponding author: Correspondence should be addressed to Feni Kontogianni, Department 16

$17 \quad$ Feni.kontogianni@port.ac.uk

\section{The Benefits of a Self-Generated Cue Mnemonic for Timeline Interviewing}

Feni Kontogianni ${ }^{\mathrm{a}^{*}}$, Lorraine Hope ${ }^{\mathrm{a}}$, Paul J. Taylor ${ }^{\mathrm{b}}$, Aldert Vrij ${ }^{\mathrm{a}}$, Fiona Gabbert ${ }^{\mathrm{c}}$

${ }^{a}$ University of Portsmouth, UK

${ }^{\mathrm{b}}$ Lancaster University, UK, and University of Twente, NL

${ }^{c}$ Goldsmiths University of London, UK

10

11

12

13

4

of Psychology, University of Portsmouth, Portsmouth, PO1 2DY. Email:

18

19

20

21 Word count: 4.754 (excluding references and figures)

22 Introduction: 1.238

23 Discussion: 835 


\section{The Benefits of a Self-Generated Cue Mnemonic for Timeline Interviewing}

26 Word count: 4.754 (excluding references and figures)

27 Introduction: 1.238

28 Discussion: 835

\section{Abstract}

Obtaining detailed accounts from individuals who have witnessed complex events under

31 challenging encoding conditions presents a difficulty for investigators. In the present research,

32 participants $(N=132)$ reported their recall of an event witnessed under full or divided attention

33 using a timeline reporting format. Extending the Timeline Technique to assess the relative

34 performance of two additional mnemonics, Self-Generated Cues (SGC) and Other-Generated

35 Cues (OGC), participants provided an account across three Timeline reporting conditions comparing the efficacy of SGC, OGC, and No Cues (control). Mock-witnesses using SGC

37 provided more correct details than mock-witnesses in the OGC or No Cues conditions, under full but not under divided attention conditions. There was no difference between cue conditions with respect to the number of errors reported across attention conditions. Findings show SGC to be a promising addition to interviewing techniques as a retrieval support mnemonic with implications

41 for applied contexts. 


\section{General Audience Summary}

48 Reliable information is critical for investigations in forensic and security settings, however

49 obtaining reliable information about complex events can be challenging. In this research, we

50 extend the Timeline Technique, which uses an innovative and interactive procedure where

51 details are reported on a physical timeline. To facilitate remembering we tested two additional

52 mnemonics, Self-Generated Cues (SGC), which witnesses produce themselves, against Other-

53 Generated Cues (OGC) which are suggested by the interviewer. One hundred and thirty-two

54 participants witnessed a multi-perpetrator theft under full or divided attention and provided an

55 account using the Timeline comparing the efficacy of SGC, OGC, and No Cues (control). Mock-

56 witnesses who used Self-Generated Cues provided more correct details than mock-witnesses in

57 the Other-Generated or No Cues conditions, with no cost to accuracy, under full but not under

58 divided attention. Promising results for SGC suggest that this mnemonic might be a useful

59 addition to current interviewing techniques.

60

61

62

63

64

65

66

67

68

69 


\section{The Benefits of a Self-Generated Cue Mnemonic for Timeline Interviewing}

Successful criminal and intelligence investigations rely on detailed and accurate information from suspects, witnesses, victims, and informants (Borum, Gelles, \& Kleinman, 2009). However, memory for experienced events is fallible and hence, sometimes inaccurate and often incomplete (Frenda, Nichols, \& Loftus, 2011; Loftus, 2003). Obtaining high-quality information can become even more difficult in cases of complex multi-perpetrator events witnessed under challenging conditions. Given that $25 \%$ of violent crimes committed by strangers involve four or more perpetrators (Office for National Statistics, 2015), and that group involvement is common in terrorist activities (Ozgul, 2016), reporting of multi-perpetrator events is relevant in both forensic and security contexts. To date, only a small body of empirical research has examined ways to improve intelligence gathering practices with calls for more focused contributions in this area (Granhag, Vrij, \& Meissner, 2014). The current research extends the Timeline Technique (Hope, Mullis, \& Gabbert, 2013), which uses an innovative reporting format to enhance retrieval of complex events, by testing the introduction of a new mnemonic, Self-Generated Cues (SGC), to facilitate recall for multi-perpetrator events witnessed under optimal (full attention) and sub-optimal conditions (divided attention).

\section{Use of Cognitive Mnemonics in Interviewing}

The use of mnemonics is already embedded in gold standard investigative interviewing practices. One example is the Mental Reinstatement of Context (MRC) of the Cognitive Interview (CI; Fisher \& Geiselman, 1992). 'Context reinstatement' capitalizes on the notion that recall increases when there is an overlap between the conditions present at encoding and at retrieval (encoding-specificity principle; Tulving \& Thomson, 1973; for a review, see Pansky, Koriat, \& Goldsmith, 2005). The administration of the MRC mnemonic, which typically elicits 
93 more correct information than free recall (e.g., Dando, Wilcock, \& Milne, 2009), involves

94 directing interviewees to think back to the surroundings, their emotional state, and their thoughts

95 around the time of the event (Memon, Wark, Bull, \& Koehnken, 1997) using pre-defined generic 96 instructions. that moderates the extent to which retrieval improves (Nairne, 2002). Cues effectively facilitate retrieval when they are distinctive in addition to satisfying the encoding-retrieval match (Tullis \& Benjamin, 2015; Watkins \& Watkins, 1975). A distinctive cue uniquely matches a memory to the exclusion of other related memories (principle of cue overload; Nairne, 2002). Therefore, to be effective, cues need to be encoded within the context of the witnessed event (encodingspecificity principle), and to offer diagnostic information identifying a single target to the

104 exclusion of others, rather than matching multiple related targets (i.e., matching but not 105 distinctive) (Goh \& Lu, 2012; Nairne, 2002). To date, research on the efficacy of cues in interviewing has mainly focused on cues generated by an interviewer, such as in the 107 administration of context reinstatement techniques. However, recent work (Wheeler, Gabbert, 108 Hope, Jones, \& Valentine, 2017) examined a new mnemonic, Self-Generated Cues (SGC) and 109 found, across two studies, that self-generated cue techniques increased reporting, with no cost to 110 accuracy, in comparison to cues generated by another witness (other-generated cues), or free 111 recall.

Self-Generated Cues are salient details that are actively generated by the individuals

113 themselves and facilitate retrieval of a target memory. When episodic information is recalled, 114 stored traces are activated and these prompt related details, thereby "spreading activation" 115 throughout an associative network (Activation Theory; Anderson, 1983). Every attempt to 
remember a detail strengthens the memory trace. The stronger the memory, the more likely it is that it will be recalled later and that it will activate associated memories (Anderson, 1983). Similarly, Anderson and Conway (1993) showed that, when asked to list event-details in free recall, participants first listed "distinctive details" (i.e., "details that really stand out and make that memory what it is", p. 1188). Then they listed other details, highly associated with those distinctive details. Thus, self-generation of distinctive cues can trigger related memories by tapping on a common theme (Anderson \& Conway, 1993; Belli, 1998). More recently, Berntsen, Staugaard, and Sørensen (2013) showed that it is possible to activate specific involuntary autobiographical memories in the lab, by manipulating the unique match between cue and item. In light of Anderson and Conway's (1993) findings, use of SGC (i.e., the most memorable details), should trigger the retrieval of related event-details while excluding unrelated details, thus satisfying both the encoding-specificity principle (Tulving \& Thomson, 1973), and the principle of cue overload (Nairne, 2002). Therefore, the present study tests the effectiveness of SGC in comparison to Other-Generated Cues and No Cues (control) across timeline reporting conditions. To maximize our test of the efficacy of SGC, in the OGC condition, we administered standard MRC instructions as a generic mnemonic (i.e. not generated by the witness). Although MRC instructions do not provide directive cues to specific aspects of an event, they suggest aspects the rememberer might focus on during retrieval. Following Wheeler et al. (2017), we predicted that use of SGC would activate unique associated memories, thus facilitating higher rates of correct recall. To examine the effectiveness of cues, and given previous research showing that accounts can be incomplete despite being accurate (Hope, Gabbert, \& Fraser, 2013; Smeets, Candel, \& Merckelbach, 2004), we also explored how the use of mnemonics affects account completeness for critical details. 


\section{Obtaining information using the Timeline Technique}

The Timeline Technique (Hope et al., 2013) uses a reporting format with a physical timeline to facilitate retrieval of multi-perpetrator events. In Hope et al. (2013), the Timeline Technique elicited more accurate information than free recall for a multi-perpetrator event and enhanced the reporting of connections between perpetrators and actions, at immediate testing and after a two weeks' delay. Importantly, instead of asking for a linear narrative of the events, the timeline format encourages witness-compatible reporting whereby interviewees can report events as they remember them, at any point of the timeline, and re-arrange details if necessary. The current study combines this reporting format with the distinctiveness of SGC to extend the Timeline Technique and evaluate a novel mnemonic.

\section{Attention and eyewitness memory}

Given the role of attention for successful encoding of witnessed events (for a review, see Pansky et al., 2005), a secondary aim was to examine recall under different encoding conditions. When witnessing a real crime, the experience of stress or physiological arousal can divert attention to aspects of the scene and/or to internal thoughts (Lane, 2006). However, laboratory studies typically use optimal conditions where participants pay full attention (FA) to events, thus possibly overestimating witnesses' memory performance (Ihlebaek, Løve, Eilertsen, \& Magnussen, 2003). Although there is some evidence of enhanced recall using cued versus free recall when attention is divided (DA) at encoding (Backman \& Nilsson, 1991), many studies have shown that DA has a robust negative effect on later remembering across stimuli (e.g., word lists, actions, pictures etc.; e.g. Craik, Govoni, Naveh-Benjamin, \& Anderson, 1996; Mulligan, 2014; Naveh-Benjamin, Kilb, \& Fisher, 2006). Using a mock-witness paradigm, Lane (2006) 
161 also found that DA at encoding resulted in lower accuracy and greater suggestibility to 162 misinformation.

Based on Activation Theory (Anderson, 1983) and given previous positive results for

164 cued versus free recall under DA (Backman \& Nilsson, 1991), we predicted that use of SGC

165 should enhance retrieval of even weakly encoded traces through the activation of memorable and 166 associated details. Although witnesses under DA conditions were expected to provide less

167 information overall, indicating poorer episodic memory, we hypothesised that witnesses in the 168 SGC condition would provide more correct information (cf. OGC and No Cue conditions) under 169 both encoding conditions.

\section{Method}

171 Participants and Design sample of 132 participants was required for a 95\% chance of detecting a large effect size (Cohen,

174 1992). A total of 135 participants were recruited through the department's participation pool and 175 through advertisements on the university campus. Participants were randomly allocated to a 3 176 (Mnemonic type: Self-Generated Cues vs Other-Generated Cues vs No Cues) x 2 (Attention at 177 encoding: Divided Attention vs Full Attention) between-subjects design. Data were excluded for 178 three participants who, respectively, did not meet the English fluency criterion, did not follow 179 the instructions in the divided attention task, and experienced an unanticipated interruption 180 during reporting. The reported analyses are based on the data for the remaining 132 (85 females; $181 \quad 18-59$ years of age; $M_{\text {age }}=25$ years, $\left.S D=8.91\right)$ participants, with 22 participants allocated per 182 group cell (SGC x FA; SGC x DA etc.).

\section{Materials}


Stimulus event. Consistent with Hope et al. (2013), the stimulus event was a multiperpetrator short film lasting $1 \mathrm{~min} 20 \mathrm{~s}$. The event showed an assault and robbery by five male perpetrators against a female victim. The film starts with three males loitering by a parked car. Two other males join them. A woman walks toward the group carrying a laptop computer bag and tries to walk past them. They surround her and one male is seen threatening her with a crowbar. Her bag is taken from her and passed between several perpetrators, while another perpetrator films the incident on his cell phone. At the end of the event, the perpetrators run away with the bag. Although there was an audio component to the video stimulus, this was mainly background traffic / outdoor noise. The content of what was said by the gang members was inaudible (in all conditions) and, as such, would not offer any additional information about the incident or actions performed.

Divided attention task. Participants allocated to the divided attention condition listened to an audio recording of a series of numbers and were instructed to respond by pressing a key when an even number was heard (adapted from Naveh-Benjamin et al., 2006) while they watched the stimulus event. The number of correct responses (hits) and reaction times to the auditory task were recorded to verify that participants attended to the distraction task as instructed. Participants who performed at lower than 50\% success at the task (from a total of 18 hits) were to be excluded from analysis, however no participants had to be excluded on this basis. As noted, one participant was excluded for not following the instructions (i.e. pressing a key to every number and not to even numbers only).

Timeline Technique. The Timeline Technique consists of three elements: (1) a physical cardboard timeline (33 in. $x 12$ in.) that has a horizontal line running at mid-point from one end of the card to the other representing the temporal context during which the event occurred; (2) 
207 blank, white, lined person description cards (5 in. x 3 in.); (3) blank yellow action cards with a

208 semi-adhesive strip on the back (3 in. $x 3$ in.) for easy removal and rearrangement on the 209 cardboard timeline.

condition were administered a version of Mental Reinstatement of Context (MRC) instructions.

212 Consistent with the standard administration of MRC, participants were instructed to think back to

213 when they witnessed the event, to think about what they could see, what they could hear, what

214 the surroundings were, and what they were thinking and feeling at the time. Participants were

215 encouraged to consider whether each prompt helped them remember other things that occurred in

216 the event. Participants were also invited to close their eyes or look at a blank wall if it helped

217 them concentrate (Dando, Wilcock, \& Milne, 2009).

Self-Generated Cues instructions. The instruction in the SGC condition was adapted from Gabbert, MacPherson, and Hope (2014). Participants were instructed to write down the first six things that they remembered seeing or thinking when viewing the event and to then focus on

221 each of these things one at a time, considering for each whether or not that memory helped them

222 remember other parts of the event. Participants were also encouraged to close their eyes or look 223 towards the wall to focus.

224 Procedure

Half of the participants watched the stimulus event while the other half watched the 226 stimulus event and simultaneously performed the auditory distraction task. All participants were 227 given the following instruction prior to watching the stimulus: "During the study, you will watch 228 a video of a crime event. Please pay attention because later you will be asked to provide an 229 account of the event." Participants in the DA condition also received the following instruction: 
230 "While you watch the video you will also listen to an audio recording of a series of numbers

231 through the headphones. Please press the "enter" key on the keyboard every time you hear an 232 even number".

233 After witnessing the event, all participants completed a 10-minute filler task (Sudoku

234 puzzle). They were then moved to a different room and were given instructions for reporting

235 their account of what happened in the event using the timeline reporting format and the

236 instructions used in Hope et al. (2013). Participants in all conditions were told to report all the

237 details about the event and the people involved that they remember, without guessing.

238 Participants were instructed on how to use the person description cards to provide information

239 about the people involved by using a new card per each individual. They were also instructed to

240 use action cards to describe any actions and information about the sequence of the events. The

241 instructions further advised that they should place all the cards on the timeline format in order,

242 with links between the individuals reported and each action to show "who did what and when".

243 Depending on condition, participants also received instructions to use Mental Reinstatement of

244 Context, or the Self-Generated Cues. Participants in the No Cues (control) condition did not

245 receive any further instructions and simply reported their account using the original Timeline

246 Technique reporting instructions. Participants were left alone in the room while providing their

247 account by completing the timeline format, although the researcher was available nearby to

248 answer any questions if necessary. Participants were not asked any questions about the witnessed

249 event by the interviewer. All participants were video-recorded while generating their accounts.

250 After participants finished providing their account, they were thanked and debriefed.

251 Coding 
The details reported by the participants on the person and action cards and placed on the

253 timeline format were then coded according to the scoring template used in Hope et al. (2013).

254 Briefly, each detail reported was identified as a Person (P), Action (A), Object (O) and Setting

255 (S) detail. A detail was scored as accurate if it was present in the stimulus event and described

256 correctly. Details that were subjective or vague were not coded for accuracy. A secondary coding

257 was conducted regarding the accuracy of attributions of the reported actions to specific actors.

258 Person-action details were scored as correct when an action was correctly attributed to a specific

259 actor (e.g., Male 3 raises the crowbar). Moreover, sequencing errors were noted when events

260 were reported in the wrong order. For instance, if ABCD is correct, in ACBD, C would be coded

261 as one sequence error as it should follow B, but B would not be counted as out of sequence too.

262 Therefore, this example reflects a total of one sequence error.

263 Finally, the reporting of critical details was coded according to the process described in

264 Smeets et al. (2004), which resulted in a list of 24 critical details [a detailed description of the

265 coding is provided in the supplementary materials]. To assess overall inter-rater reliability, 20

266 interviews were randomly selected and coded independently by a rater blind to experimental

267 conditions. Inter-rater reliability was high, ICC $=.98,95 \%$ CI $[.967, .988]$ across coding

268 categories.

Results

Bonferroni-corrected pairwise comparisons were conducted for all interactions. In the

271 interests of parsimony, we only report pairwise comparisons where they indicate significant

272 differences (even for non-significant interactions). Where Bonferroni-corrected pairwise

273 comparisons are not significant (and therefore do not aid interpretation beyond the non-

274 significant interactions), they are not reported. 


\section{Reporting of Correct Details}

A between-subjects ANOVA showed a significant main effect of Cues, $F(2,126)=4.39$,

$277 p=.014, \omega^{2}=.049$, for the number of correct details reported. Post hoc tests showed that, across

278 attention conditions, more correct details were reported in the Self-Generated Cues condition

279 than in the No Cues condition $(p=.012)$. The number of correct details reported in the Other-

280 Generated Cues condition did not differ from the number of correct details reported in the Self-

281 Generated Cues $(p=.241)$ and No Cues $(p=.718)$ conditions. There was also a main effect of

282 Attention, $F(1,126)=24.78, p<.001, \omega^{2}=.156$, with significantly more correct details reported

283 in the Full attention condition than in the Divided attention condition. The interaction between

284 Attention and Cues was not significant, $F(2,126)=2.23, p=.111, \omega^{2}=.018$. Bonferroni-

285 corrected pairwise comparisons showed that more correct details were reported in the Self-

286 Generated Cues condition than in either the Other-Generated Cues $(p=.046)$ or No Cues $(p=$

$287.002)$ condition, under full attention, while there was no difference between conditions under

288 divided attention $(p=1.00)$. Results for the number of incorrect details are reported in

289 supplementary materials.

290 The effect of cues on the mean number of correct details reported within Full and

291 Divided attention conditions are presented in Figure 1. 


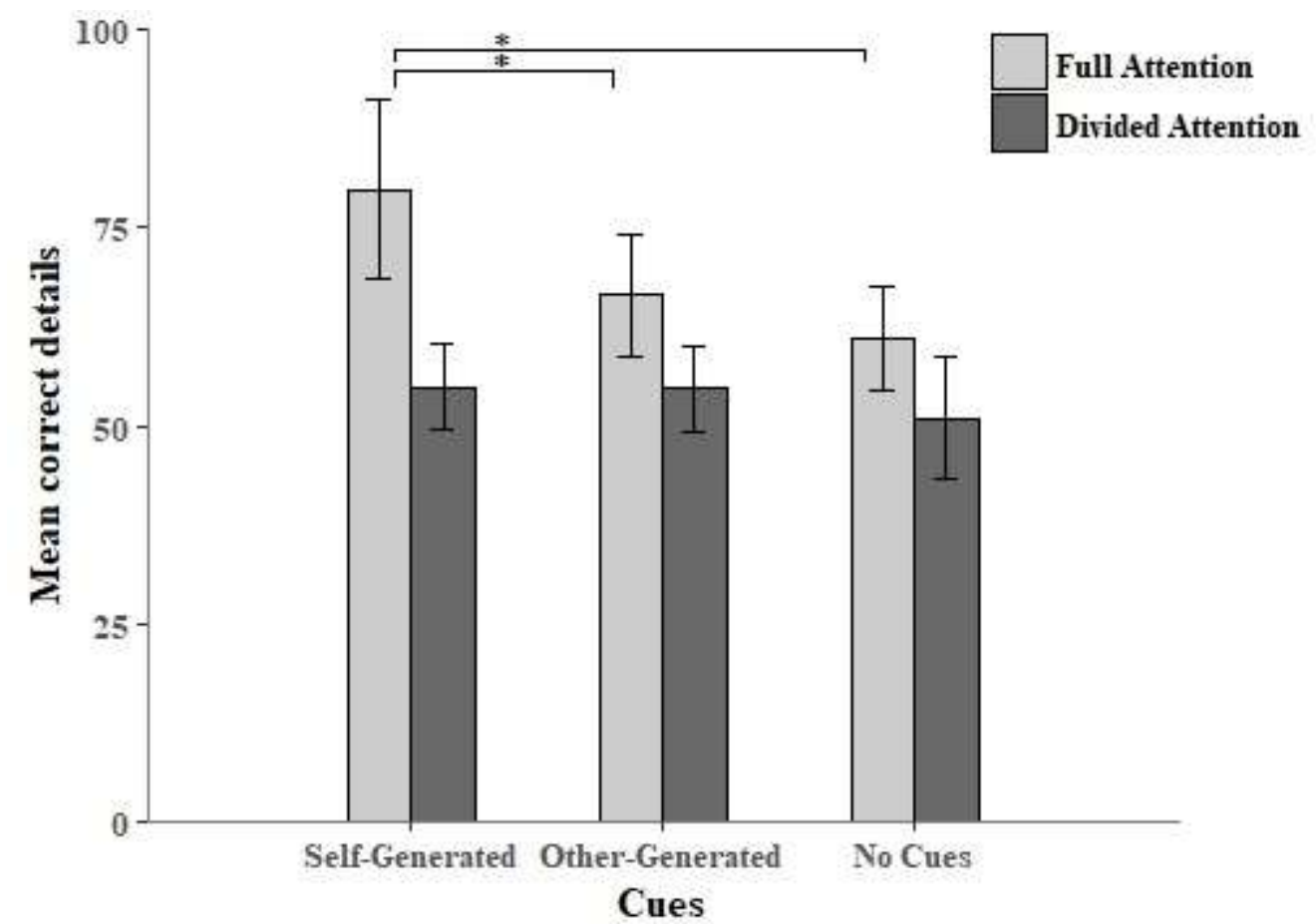

293 Figure 1. Mean number of correct details reported as a function of cues (Self-Generated Cues vs 294 Other-Generated Cues vs No Cues) within Full and Divided attention conditions. Error bars 295 represent \pm 1.96 standard errors (95\% confidence intervals). Asterisks indicate significant 296 differences between cue conditions, ${ }^{*} p<.05$.

297 Accuracy Rate of Reported Details

Accuracy rate was calculated by dividing the number of correct details by the sum of 299 both correct and incorrect details (total number of items) to obtain the proportion of accurate 300 reported information. Levene's test was significant $(p=.004)$. A boxplot showed that the 301 distribution was not symmetrical but negatively skewed with two outliers who had particularly 302 low scores. However, given the overall robustness of the test, no action was taken. Analysis 303 revealed a significant main effect of Attention, $F(1,126)=10.37, p=.002, \omega^{2}=.068$, with 304 higher accuracy rates in the Full (cf. Divided) attention condition. There was also a main effect 305 of Cues, $F(2,126)=3.43, p=.035, \omega^{2}=.036$, on accuracy rates. Post hoc tests with a Bonferroni 
306 adjustment showed that across attention conditions, there was no significant difference between

307 the accuracy rate in the Self-Generated Cues condition and the accuracy rate in the Other-

308 Generated Cues $(p=1.00)$ or No Cues conditions $(p=.188)$. However, the accuracy rate in the

309 Other-Generated Cues condition was significantly higher than the rate in the No Cues $(p=.039)$

310 condition. The interaction was not significant, $F(2,126)=.63, p=.536, \omega^{2}=-.005$. Bonferroni-

311 corrected pairwise comparisons showed that there was no significant difference in accuracy rates

312 between Self-Generated Cues and Other-Generated Cues conditions ( $p=1.00)$, Self-Generated

313 Cues and No Cues conditions $(p=.783)$ or Other-Generated Cues and No Cues conditions $(p=$

314 .932) under full attention. Under divided attention, there was a significantly higher accuracy rate

315 in the Other-Generated Cues condition compared to the No Cues condition $(p=.036)$, however

316 there was no significant difference between accuracy rates in the Self-Generated Cues and Other-

317 Generated Cues conditions $(p=.388)$

\section{Attribution of Actions}

With respect to correct person-action details, there was a significant main effect of

320 Attention, $F(1,126)=8.94, p=.003, \omega^{2}=.058$, but not of Cues, $F(2,126)=.003, p=.997, \omega^{2}=$

$321-.007$. The interaction between Attention and Cues was not significant, $F(2,126)=.21, p=.814$,

$322 \omega^{2}=-.012$. Results for incorrect person-action details are reported in supplementary materials.

323 The main effects for correct person-action details are presented in Figure 2. 


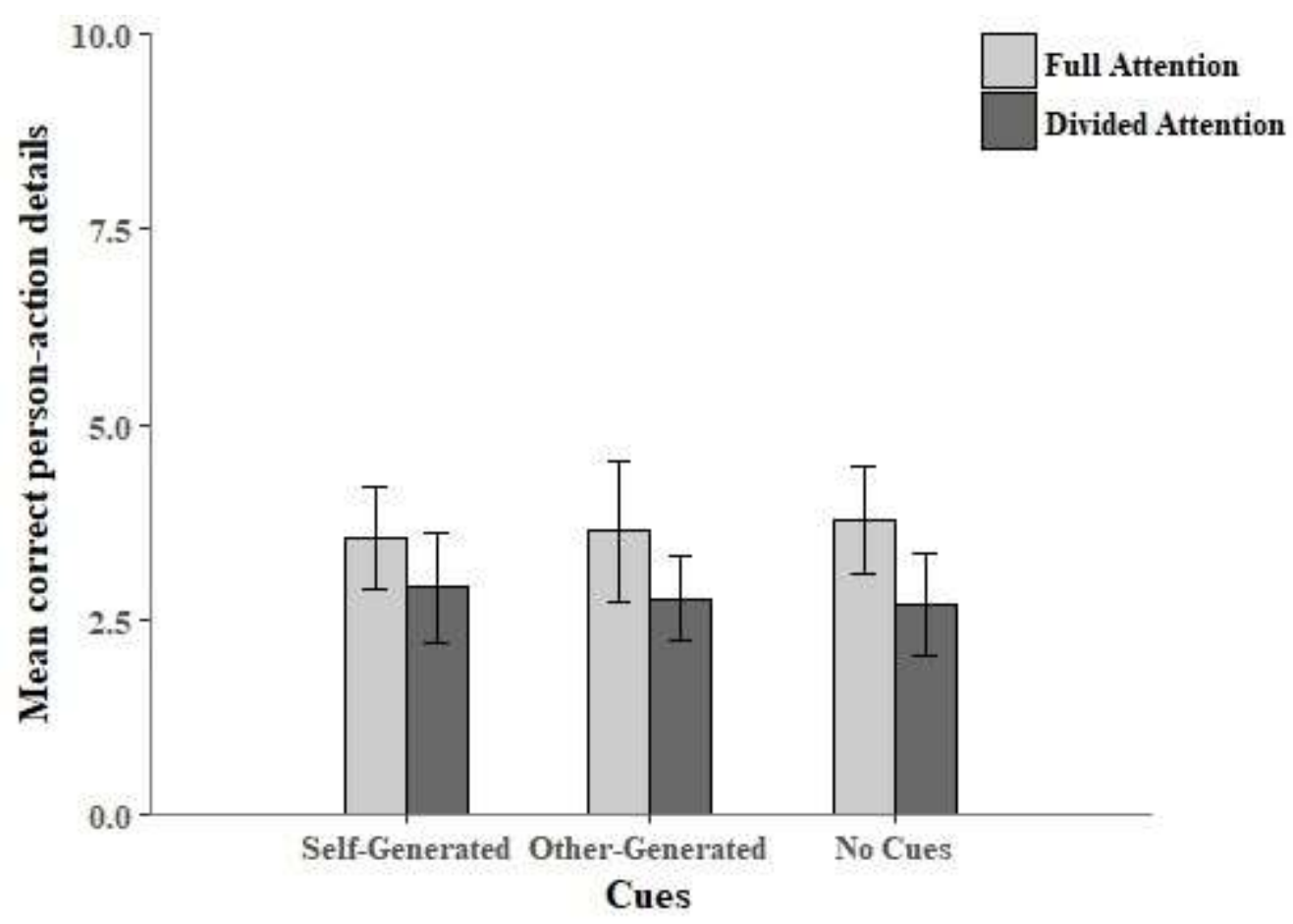

Figure 2. Mean number of correct person-action details as a function of cues (Self-Generated

327 Cues vs Other-Generated Cues vs No Cues) and attention (Full vs Divided attention). Error bars represent \pm 1.96 standard errors (95\% confidence intervals).

\section{Accuracy Rate of Person-Action Details}

With respect to the accuracy rate of person-action details, there was no significant main

331 effect of Attention, $F(1,126)=2.08, p=.152, \omega^{2}=.008$, or Cues, $F(2,126)=.10, p=.910, \omega^{2}=$ $332-.014$. The interaction was also not significant, $F(2,126)=2.77, p=.066, \omega^{2}=.026$.

\section{Sequence errors}

There was a main effect of Attention $F(1,126)=4.19, p=.043, \omega^{2}=.024$, but not of

335 Cues, $F(2,126)=.029, p=.971, \omega^{2}=-.015$ on the total number of sequence errors reported by 336 participants. The interaction between Attention and Cues for the total number of sequence errors 337 reported by participants was significant, $F(2,126)=3.75, p=.026, \omega^{2}=.040$. Pairwise 
338 comparisons showed that there were significantly more sequence errors made with the use of

339 Other-Generated Cues under Full attention $(M=.55, \mathrm{SE}=.05)$ compared to the Divided

340 attention condition $(M=.05, \mathrm{SE}=.02)(p=.001)$. However, there was no difference between

341 attention conditions for the number of sequence errors made in the Self-Generated Cues $(p=$

$342.377)$ and No Cues $(p=.556)$ conditions. Levene's test was significant for the analysis of

343 sequence errors $(p<.001)$. Since the values in the reporting of sequence errors were overall very

344 low $(M=.30, \mathrm{SD}=.52)$, no action was taken to recover the assumptions violation. Instead,

345 emphasis was given to the fact that the overall mean number of sequence errors was low.

Results for the effects of Cues and Attention on the reporting of critical details and detail

347 type (person, action, object, setting) are reported in the supplementary materials.

\section{Discussion}

349 We tested the effectiveness of cognitive mnemonics used in conjunction with the Timeline

350 Technique under full and divided attention. As predicted, mock-witnesses who used Self-

351 Generated Cues (SGC) reported more correct details than mock-witnesses in Other-Generated

352 and No Cue conditions, at no cost to accuracy. However, this enhanced performance with SGC

353 was only observed under full attention. Participants under divided attention consistently reported

354 less correct information than those under full attention, and there was no effect of cues under 355 divided attention.

The apparent lack of benefit of SGC under divided attention is noteworthy. The sizeable

357 main effect of the divided attention task across cue conditions suggests that performing a

358 secondary task significantly challenged attentional processes and likely drew participants'

359 attention away from the target event, thus restricting encoding and retrieval (see also Marsh et

360 al., 2017, for a similar DA effect when participants were instructed to ignore distractions). These 
361 findings are consistent with literature on the powerful effect of divided attention on remembering

362 (e.g., Craik et al., 1996) and, although it is not surprising that our task restricted encoding (as

363 intended), it is possible that the to-be-remembered information was not stored from the outset,

364 thus hindering retrieval despite the additional support of cues. Another possibility is that the

365 SGC manipulation was simply not powerful enough to access weakly encoded memories. Given

366 that research on the effectiveness of memory-enhancing techniques under sub-optimal encoding

367 conditions is limited, more research is needed to determine the most likely explanation. Research

368 should also examine the effectiveness of SGC possibly with more naturalistic divided attention

369 measures, such as using a smartphone or conversing (e.g. Marsh et al., 2017), to delineate the

370 limitations of the use of cues.

371 Nevertheless, mock-witnesses reported more correct information under full attention with

372 SGC than with OGC. Possibly, the use of SGC facilitated retrieval more effectively across the

373 whole event by activating the "stronger" memories (Anderson, 1983) that distinctively identify

374 associated targets (Nairne, 2002). It is also possible that initially identifying six event-details and

375 processing them further might contribute to the SGC advantage. By comparison, Other-

376 Generated Cues, administered here in the form of generic context-retrieval cues, failed to

377 activate as many event-details. Further research is needed to increase understanding about the

378 underlying mechanisms of SGC relative to more generic cues (e.g., OGC).

379 Another caveat to our finding of superior performance by SGC is that there was no effect

380 of cues on the reporting of critical details. Overall, only $50 \%$ of the critical details identified by

381 legal professionals were reported across conditions, suggesting that even highly accurate and

382 detailed accounts can be lacking in information relevant to investigators (see Hope et al., 2013;

383 Smeets et al., 2004). Notably, most of these critical details related to specific details of the 
384 assault. It is possible that mock-witnesses did not appreciate the level of detail required or that, 385 given the brevity of the event, such details were poorly encoded or simply not salient for 386 participants and, therefore, not prompted by the SGC. Future research might examine whether 387 follow-up questioning facilitates the reporting of such details. attributions of actions. Accounts of witnesses using SGC or OGC did not include more personaction details than accounts of witnesses in the control condition, who only used the Timeline

391 Technique. Therefore, the use of mnemonics did not increase the reporting of person-action 392 details. Thus, features of the Timeline Technique (likely the use of different person and action 393 cards and the instruction to show "who did what when") possibly drove the reporting of person394 actions details. Indeed, in Hope et al. (2013) reporting of person-action details did not differ 395 between participants when using the Timeline Technique to participants using person and action 396 cards only (Experiment 2). Given that SGC increased retrieval of correct information overall, but 397 did not improve the reporting of person-action details compared to use of the timeline alone, it 398 may be worth exploring whether SGC and timeline capitalize on different retrieval processes to 399 access different types of information.

400 Although our expectations about the benefit of SGC across encoding conditions were not 401 fully met, the results of SGC in the full attention condition are promising. Notably for applied 402 contexts where person descriptions are valuable in investigations (Brown, Lloyd-Jones, \& 403 Robinson, 2008; Gabbert \& Brown, 2015), witnesses who used SGC reported more person 404 details compared to other conditions, with person details being reported to a greater extent than 405 any other details. 
Current findings suggest that, when attention at encoding has not been compromised,

407 Self-Generated Cues may be a useful addition to interviewing techniques as a retrieval support

408 mnemonic that promotes witness-led interviewing. In intelligence gathering, interviewers may be

409 unaware of what information interviewees possess and what is memorable to each interviewee.

410 Accordingly, the use of SGC may support the interviewing process by facilitating an open-

411 ended, largely self-administered report. Not only does this approach allow witnesses to report

412 event-details in their own words; it also limits the potential for use of inappropriate or leading

413 questions.

Author contributions

415 First and second author conceived the research idea. First author designed, conducted, and 416 analysed the research and wrote the research paper. Second, third, fourth, and fifth authors

417 provided feedback on the research and reviews on the research paper.

Acknowledgements

419 We thank Shiri Portnoy for her contribution in assessing inter-rater reliability, Nicole

420 Breakspeare and Oliver Pyke for help with data collection, and Marc Baker for his comments on 421 an earlier version of this paper. This research forms part of the doctoral work of the first author 422 who is in receipt of a doctoral studentship funded by the Centre for Research and Evidence on 423 Security Threats (ESRC Award: ES/N009614/1).

\section{References}

425 Anderson, J. R. (1983). A spreading activation theory of memory. Journal of Verbal Learning and Verbal Behavior, 22(3), 261-295. doi:10.1016/S0022-5371(83)90201-3 
427

428

429

430

431

432

433

434

435

436

437

438

439

440

441

442

443

444

445

446

447

448

Anderson, S. J., \& Conway, M. A. (1993). Investigating the structure of autobiographical memories. Journal of Experimental Psychology: Learning, Memory, and Cognition, 19(5), 1178-1196. doi:10.1037/0278-7393.19.5.1178

BÄckman, L., \& Nilsson, L. G. (1991). Effects of divided attention on free and cued recall of verbal events and action events. Bulletin of the Psychonomic Society, 29(1), 51-54. doi:10.3758/BF03334767

Belli, R. F. (1998). The structure of autobiographical memory and the event history calendar: Potential improvements in the quality of retrospective reports in surveys. Memory, 6(4), 383-406. doi:10.1080/741942610

Berntsen, D., Staugaard, S. R., \& Sørensen, L. M. T. (2013). Why am I remembering this now? Predicting the occurrence of involuntary (spontaneous) episodic memories. Journal of Experimental Psychology: General, 142(2), 426-444. doi:10.1037/a0029128

Borum, R., Gelles, M., \& Kleinman, S. (2009). Interview and interrogation: A perspective and update from the USA. In R. Milne, S. Savage, \& T. Williamson (Eds.) International Developments in Investigative Interviewing (pp. 111-128). Cullompton, UK: Willan Publishing.

Brown, C., Lloyd-Jones, T. J., \& Robinson, M. (2008). Eliciting person descriptions from eyewitnesses: A survey of police perceptions of eyewitness performance and reported use of interview techniques. European Journal of Cognitive Psychology, 20(3), 529-560. doi:10.1080/09541440701728474

Cohen, J. (1992). A power primer. Psychological bulletin, 112(1), 155-159. http://dx.doi.org/10.1037/0033-2909.112.1.155 
449 Craik, F. I., Govoni, R., Naveh-Benjamin, M., \& Anderson, N. D. (1996). The effects of divided

450

451

452

453

454

455

456

457

458

459

460

461

462

463

464

465

466

467

468

469

470

471

attention on encoding and retrieval processes in human memory. Journal of Experimental Psychology: General, 125(2), 159-180. doi:10.1037/0096-3445.125.2.159

Cumming, G. (2013). Understanding the new statistics: Effect sizes, confidence intervals, and meta-analysis. New York, NY, USA: Routledge, USA.

Dando, C., Wilcock, R., \& Milne, R. (2009). The cognitive interview: The efficacy of a modified mental reinstatement of context procedure for frontline police investigators. Applied Cognitive Psychology, 23(1), 138-147. doi:10.1002/acp.1451

Faul, F., Erdfelder, E., Lang, A. G., \& Buchner, A. (2007). G* Power 3: A flexible statistical power analysis program for the social, behavioral, and biomedical sciences. Behavior research methods, 39(2), 175-191. doi:10.3758/BF03193146

Fisher, R., \& Geiselman, R. (1992). Memory-enhancing techniques for investigative Interviewing: The cognitive interview. Springfield, IL, USA: Charles C. Thomas.

Frenda, S. J., Nichols, R. M., \& Loftus, E. F. (2011). Current issues and advances in misinformation research. Current Directions in Psychological Science, 20(1), 20 23. https://doi.org/10.1177/0963721410396620

Gabbert, F. \& Brown, C. (2015). Interviewing for face identification. In T. Valentine, \& J. P. Davis, (Eds.), Forensic Facial Identification: Theory and Practice of Identification from Eyewitnesses, Composites and CCTV. Chichester, UK: Wiley-Blackwell.

Gabbert, F., MacPherson, I., \& Hope, L. (2014). Adding a new mnemonic to the Cognitive Interview. Paper presented at IIIRG Conference, Lausanne, 2014.

Goh, W. D., \& Lu, S. H. (2012). Testing the myth of the encoding-retrieval match. Memory \& cognition, 40(1), 28-39. doi:10.3758/s13421-011-0133-9 
472 Granhag, P. A., Vrij, A., \& Meissner, C. A. (2014). Information gathering in law enforcement

473

474

475

476

477

478

479

480

481

482

483

484

485

486

487

488

489

490

491

492 and intelligence settings: Advancing theory and practice. Applied Cognitive Psychology, 28(6), 815-816. doi:10.1002/acp.3093

Hope, L., Gabbert, F., \& Fraser, J. (2013). Postincident conferring by law enforcement officers: Determining the impact of team discussions on statement content, accuracy, and officer beliefs. Law and Human Behavior, 37(2), 117-127. doi:10.1037/1hb0000019

Hope, L., Mullis, R., \& Gabbert, F. (2013). Who? What? When? Using a timeline technique to facilitate recall of a complex event. Journal of Applied Research in Memory and Cognition, 2(1), 20-24. doi:10.1016/j.jarmac.2013.01.002

Ihlebæk, C., Løve, T., Erik Eilertsen, D., \& Magnussen, S. (2003). Memory for a staged criminal event witnessed live and on video. Memory, 11(3), 319-327. $\underline{\text { doi: } 10.1080 / 09658210244000018}$

Lane, S. M. (2006), Dividing attention during a witnessed event increases eyewitness suggestibility. Applied Cognitive Psychology, 20(2), 199-212. doi:10.1002/acp.1177

Loftus, E. (2003). Our changeable memories: Legal and practical implications. Nature Reviews Neuroscience, 4(3), 231-234. doi:10.1038/nrn1054

Marsh, J. E., Patel, K., Labonté, K., Threadgold, E., Skelton, F. C., Fodarella, C., ... \& Vachon, F. (2017). Chatting in the face of the eyewitness: The impact of extraneous cell-phone conversation on memory for a perpetrator. Canadian Journal of Experimental Psychology/Revue canadienne de psychologie expérimentale, 71(3), 183-190. 
493 Memon, A., Wark, L., Bull, R., \& Koehnken, G. (1997). Isolating the effects of the cognitive

494

495

496

497

498

499

500

501

502

503

504

505

506

507

508

509

510

511

512

513

514 interview techniques. British Journal of Psychology, 88(2), 179-197. doi:10.1111/j.20448295.1997.tb02629.x

Mulligan, N. W. (2014). Memory for pictures and actions. In T. J. Perfect, \& D. S. Lindsay (Eds.), The SAGE handbook of applied memory, (pp. 20-36). London, UK: Sage.

Nairne, J. S. (2002). The myth of the encoding-retrieval match. Memory, 10(5-6), 389-395. doi:10.1080/09658210244000216

Naveh-Benjamin, M., Kilb, A., \& Fisher, T. (2006). Concurrent task effects on memory encoding and retrieval: Further support for an asymmetry. Memory \& Cognition, 34(1), 90-101. doi:10.3758/BF03193389

Ozgul, F. (2016). Analysis of topologies and key players in terrorist networks. Socio-Economic Planning Sciences, 56, 40-54. doi:10.1016/j.seps.2016.07.002

Office for National Statistics (2015). Focus on Violent Crime and Sexual Offences: Year ending March 2015. Retrieved from: https://www.ons.gov.uk/peoplepopulationandcommunity/crimeandjustice/compendium/f ocusonviolentcrimeandsexualoffences/yearendingmarch2015

Pansky, A., Koriat, A., \& Goldsmith, M. (2005). Eyewitness recall and testimony. In N. Brewer, \& K. D. Williams (Eds.), Psychology and law: An empirical perspective, (pp. 93-150). New York, NY, USA: Guilford Press.

Smeets, T., Candel, I., \& Merckelbach, H. (2004). Accuracy, completeness, and consistency of emotional memories. American Journal of Psychology, 117(4), 595-609. doi:10.2307/4148994 
515 Tullis, J. G., \& Benjamin, A. S. (2015). Cue generation: How learners flexibly support future

516

517

518

519

520

521

522

523

524

525

526

527

528

529

530

531

532

534

535

536

537

533 retrieval. Memory \& Cognition, 43(6), 922-938. doi:10.3758/s13421-015-0517-3

Tulving, E., \& Thomson, D. M. (1973). Encoding specificity and retrieval processes in episodic memory. Psychological Review, 80(5), 352-373. doi:10.1037/h0020071

Watkins, O. C., \& Watkins, M. J. (1975). Buildup of proactive inhibition as a cue-overload effect. Journal of Experimental Psychology: Human Learning and Memory, 1(4), 442452. doi:10.1037/0278-7393.1.4.442

Wheeler, R. L., Gabbert, F., Hope, L., \& Jones, S. (2017, January). Evaluating a Self-Generated Cue Mnemonic to Enhance Eyewitness Retrieval. Paper presented at SARMAC conference, Sydney, Australia.

5

6

7

8

29

30

31

35

36


539 In this Supplementary Materials section, we provide information about coding and analyses for 540 variables which are conventional in this research area (e.g. reporting of incorrect details) but 541 which lie outside our main hypotheses.

542

543 1. Critical Details Coding

544 Prior to data collection, six legal professionals viewed the stimulus event and

545 independently provided a list of details that they considered critical to pursue an investigation of 546 the assault and relevant legal charges. Details mentioned by at least four of the six legal

547 professionals were included in a final list of 24 critical details. Accounts were then coded for the 548 reporting of these critical details. To calculate a completeness rate for critical details, the total of 549 reported critical details was divided by 24 (i.e. the maximum number of critical details). Higher 550 scores indicated higher levels of completeness.

\section{Supplementary Results (main results reported in manuscript)}

\section{Reporting of Incorrect Details}

There was no significant main effect of Cues, $F(2,126)=1.10, p=.337, \omega^{2}=.001$, or

555 Attention, $F(1,126)=.08, p=.777, \omega^{2}=-.007$, on the total number of incorrect details reported.

556 The interaction between Attention and Cues was not significant, $F(2,126)=.23, p=.793, \omega^{2}=-$

557 .012. Means for incorrect details reported as a function of cue and attention conditions are

558 presented in Table 1.

559 Table 1. Mean number (SE) of incorrect details by cues (Self-Generated Cues, Other-Generated 560 Cues, No Cues) and attention (Full and Divided). 


\begin{tabular}{|c|c|c|c|c|c|c|}
\hline \multirow[b]{3}{*}{ Attention } & \multirow[b]{3}{*}{$M(S E)$} & \multicolumn{5}{|c|}{ Incorrect details } \\
\hline & & \multicolumn{2}{|l|}{ SGC } & \multicolumn{2}{|l|}{ OGC } & \multirow{2}{*}{$\begin{array}{l}\text { NC } \\
95 \% \mathrm{CI}\end{array}$} \\
\hline & & $95 \% \mathrm{CI}$ & $M(S E)$ & $95 \% \mathrm{CI}$ & $M(S E)$ & \\
\hline Full & $9.9(0.5)$ & {$[7.5,12.3]$} & $9.3(0.7)$ & {$[6.6,12.4]$} & $11(0.6)$ & {$[7.6,13.6]$} \\
\hline Divided & $10.1(0.4)$ & {$[8.3,11.9]$} & $8.7(0.3)$ & {$[7.1,10.4]$} & $11.6(0.7)$ & {$[8.6,14.7]$} \\
\hline
\end{tabular}

\section{Reporting of Incorrect Action Attributions}

There was no effect of either Attention, $F(1,126)=0, p=1.00, \omega^{2}=-.008$, or Cues, $F(2,126)=.74, p=.479, \omega^{2}=-.004$, on the total number of incorrect person-action details. No significant interaction emerged between Cues and Attention, $F(2,126)=2.01, p=.138, \omega^{2}=$

567.015. Means for incorrect person-action details reported as a function of cue and attention 568 conditions are presented in Table 2.

569 Table 2. Mean number (SE) of incorrect person-action details by cues (Self-Generated Cues, Other-Generated cues, No Cues) and attention (Full and Divided).

\begin{tabular}{|c|c|c|c|c|c|c|}
\hline \multirow[b]{3}{*}{ Attention } & \multirow[b]{3}{*}{$M(S E)$} & \multicolumn{5}{|c|}{ Incorrect Person-Action details } \\
\hline & & \multicolumn{2}{|l|}{ SGC } & \multicolumn{2}{|l|}{ OGC } & \multirow{2}{*}{$\begin{array}{l}\mathrm{NC} \\
95 \% \mathrm{CI}\end{array}$} \\
\hline & & $95 \% \mathrm{CI}$ & $M(S E)$ & $95 \% \mathrm{CI}$ & $M(S E)$ & \\
\hline Full & $0.86(0.1)$ & {$[0.53,1.21]$} & $1.5(0.1)$ & {$[0.91,2.32]$} & $1.05(0.1)$ & {$[0.56,1.56]$} \\
\hline
\end{tabular}


Divided $\quad 1.05(0.1) \quad[0.58,1.54] 0.91(0.1) \quad[0.54,1.35] \quad 1.45(0.1) \quad[0.95,2.00]$

571

572

573

574

575

576

577

578

579

580

581

582

Generated cues, No Cues) and attention (Full and Divided).

\section{Reporting of Critical Details} attention conditions are presented in Table 3.

The mean number of reported critical details across conditions was $12(S D=2.9)$ out of a total of 24 details. There was a significant main effect of Attention on the total number of reported crime-related details, $F(1,126)=28.00, p<.001, \omega^{2}=.174$, but there was no main effect of Cues, $F(2,126)=.06, p=.940, \omega^{2}=-.014$. No significant Attention by Cue interactions emerged for reported critical details, $F(2,126)=.51, p=.600, \omega^{2}=-.008$. Finally, there was a significant main effect of Attention, $F(1,126)=28.48, p<.001, \omega^{2}=.176$, but not Cues, $F(2,126)=.05, p=.954, \omega^{2}=0.014$, on the rate of completeness of participants' accounts. The interaction between Attention and Cues was not significant for the rate of completeness, $F(2,126)=.44, p=.647, \omega^{2}=-.009$. Means for reported critical details as a function of cue and

Table 3. Mean number (SE) of reported critical details by cues (Self-Generated Cues, Other-

\begin{tabular}{|c|c|c|c|c|c|c|}
\hline \multirow[b]{3}{*}{ Attention } & \multicolumn{6}{|c|}{ Reported details } \\
\hline & & SGC & & OGC & & $\mathrm{NC}$ \\
\hline & $M(S E)$ & $95 \% \mathrm{CI}$ & $M(S E)$ & $95 \% \mathrm{CI}$ & $M(S E)$ & $95 \% \mathrm{CI}$ \\
\hline Full & $13.1(0.2)$ & {$[12.2,14.1]$} & $12.7(0.2)$ & {$[11.8,13.5]$} & $12.9(0.2)$ & {$[11.9,14]$} \\
\hline Divided & $10.3(0.2)$ & {$[9.3,11.6]$} & $10.9(0.3)$ & {$[9.6,12]$} & $10.2(0.3)$ & {$[8.8,11.6]$} \\
\hline
\end{tabular}




\section{Type of Details Reported}

There was a main effect on the total number of person details for Attention, $F(1,126)=$ $14.55, p<.001, \omega^{2}=.095$, with more person details reported under full than divided attention. There was also a main effect of Cues, $F(2,126)=4.91, p=.009, \omega^{2}=.057$. Post-hoc tests showed that more person details were reported overall with SGC than with No Cues $(p=.011)$, but not compared to the Other-Generated Cues condition $(p=.061)$. There was also no significant difference in the number of person details reported in the Other-Generated Cues condition in comparison to the No Cues condition $(p=1.00)$. No significant interaction emerged for the total number of person details, $F(2,126)=1.40, p=.251, \omega^{2}=.006$. Bonferroni-corrected pairwise comparisons revealed that the use of Self-Generated Cues led to the reporting of more person details comparing to the use of Other-Generated Cues $(p=.039)$ and of No Cues $(p=$ .005), under the Full attention condition. However, there was no difference between cues under Divided attention conditions $(p>.05)$.

There was a main effect of Attention, $F(1,126)=8.64, p=.004, \omega^{2}=.056$, but not of Cues, $F(2,126)=.24, p=.788, \omega^{2}=-0.011$, on the total number of object details reported. There was no significant interaction between Cues and Attention, $F(2,126)=1.32, p=.272, \omega^{2}=.005$. Similarly, there was a main effect of Attention, $F(1,126)=15.57, p<.001, \omega^{2}=.102$, but not of Cues, $F(2,126)=.03, p=.966, \omega^{2}=-0.015$, on the total number of action details reported. The interaction between Attention and Cues was not significant, $F(2,126)=1.01, p=.366, \omega^{2}=.000$. Levene's test was significant for the analysis of action details $(p=.03)$. Finally, there was no effect of Attention, $F(1,126)=.62, p=.434, \omega^{2}=-.003$ or Cue, $F(2,126)=2.86, p=.061, \omega^{2}=$ .028 , on the total number of setting details reported. Levene's test was significant $(p=.005)$. No significant interaction emerged for the reporting of setting details, $F(2,126)=.70, p=.499, \omega^{2}=$ 
$610-.005$. Boxplots were used to explore the distribution for the total number of both action and

611 setting details. For action details, the distribution was symmetrical however there were seven

612 outliers representing participants who reported a high number of action details. For setting

613 details, the distribution was not symmetrical but positively skewed with three outliers who

614 reported a high number of setting details. Given the low number particularly regarding setting

615 details $(M=6.88, S D=3.58)$, and the lack of significant results for both type of details, no

616 action was taken due to the Levene's test being significant. The effect of cues on the mean

617 number of person details within Full and Divided attention conditions are presented in Figure 1.

618 Means for action, object and setting details reported within both attention conditions are

619 presented in Tables $4 \mathrm{a}$ and $4 \mathrm{~b}$.

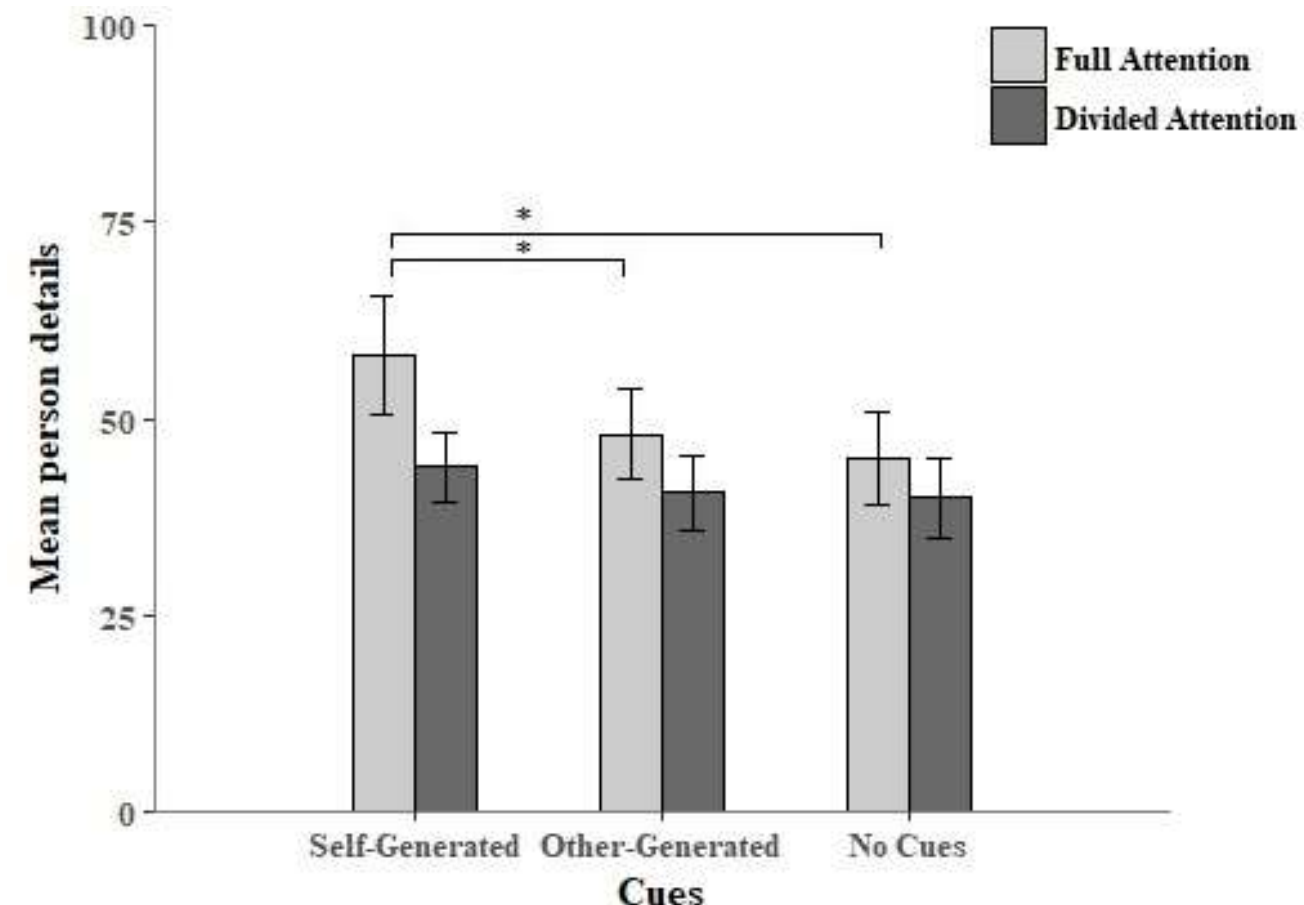

620

Figure 1. Mean number of person details as a function of cues (Self-Generated Cues vs Other-

622 Generated Cues vs No Cues) within Full and Divided attention conditions. Error bars represent \pm 
6231.96 standard errors (95\% confidence intervals). Asterisks indicate significant differences

624 between cue conditions, $* p<.05$.

625 Table 4a. Mean (SE) number of action, object and setting details by cues (Self-Generated Cues, 626 Other-Generated Cues, No Cues) under Full attention.

Full Attention

\begin{tabular}{ccccccc}
\cline { 3 - 6 } & & \multicolumn{2}{c}{ SGC } & \multicolumn{2}{c}{ OGC } & \multicolumn{2}{c}{ NC } \\
\hline $\begin{array}{c}\text { Details } \\
\text { type }\end{array}$ & $M(S E)$ & $95 \% \mathrm{CI}$ & $M(S E)$ & $95 \% \mathrm{CI}$ & $M(S E)$ & $95 \% \mathrm{CI}$ \\
\hline Action & $18.4(0.6)$ & {$[15.5,21.3]$} & $18.1(0.8)$ & {$[14.6,21.6]$} & $16.3(0.6)$ & {$[13.8,19.1]$} \\
& & & & & & \\
Object & $10(0.3)$ & {$[8.7,11.3]$} & $9.2(0.3)$ & {$[7.8,10.7]$} & $9.2(0.3)$ & {$[8.1,10.4]$} \\
& & & & & & \\
Setting & $8.4(0.4)$ & {$[6.3,10.4]$} & $7.2(0.4)$ & {$[5.5,9.1]$} & $5.8(0.2)$ & {$[4.9,6.7]$}
\end{tabular}

627

628

Table 4b. Mean (SE) number of action, object and setting details by cues (Self-Generated Cues,

Other-Generated Cues, No Cues) under Divided attention.

\begin{tabular}{lcccccc}
\hline & \multicolumn{5}{c}{ Divided Attention } \\
\cline { 2 - 7 } & \multicolumn{2}{c}{ SGC } & \multicolumn{2}{c}{ OGC } & \\
\hline Details & $M(S E)$ & $95 \% \mathrm{CI}$ & $M(S E)$ & $95 \% \mathrm{CI}$ & $\mathrm{M}(\mathrm{SE})$ & $95 \% \mathrm{CI}$ \\
type & & & & & \\
\hline Action & $12.3(0.4)$ & {$[10.5,14.2]$} & $12.9(0.4)$ & {$[10.8,14.9]$} & $10.5)$ & {$[11.6,16.5]$} \\
Object & $7.2(0.2)$ & {$[6,8.4]$} & $8.6(0.2)$ & {$[7.5,9.8]$} & $7.7(0.4)$ & {$[6,9.5]$} \\
Setting & $7.3(0.3)$ & {$[6,8.5]$} & $6.3(0.2)$ & {$[5.3,7.3]$} & $6.3(0.3)$ & {$[4.9,7.9]$} \\
\hline
\end{tabular}

